\title{
A EXENTERAÇÃO PÉLVICA NO TRATAMENTO DO CÂNCER DE RETO ESTÁDIO T4: experiência de 15 casos operados
}

\author{
Sergio Renato Pais COSTA, Ricardo César Pinto ANTUNES, Raphael Paulo de PAULA, \\ Miguel Ângelo PEDROSO, José Francisco de Mattos FARAH e Renato Arioni LUPINACCI
}

RESUMO - Racional - A exenteração pélvica tem sido a melhor opção terapêutica radical para o tratamento dos tumores de reto T4. No entanto, essa operação ainda permanece com mortalidade significante e alta morbidade. Objetivo - Relatar série de 15 casos de exenteração pélvica para tumores de reto T4, analisando a morbidade, mortalidade e sobrevida dos pacientes. Métodos - Foram estudados 15 pacientes com câncer de reto T4 no Serviço de Cirurgia Geral - Oncocirurgia do Hospital do Servidor Publico Estadual de São Paulo, SP, submetidos a exenteração pélvica no período de 1998 e 2006. Sete eram do sexo masculino enquanto oito eram do sexo feminino, com média de idade de 65 anos. Todos apresentavam sintomas incapacitantes. As operações foram: exenteração infra-elevadora $(n=6)$, exenteração supra-elevadora $(n=4)$, exenteração posterior $(n=3)$ e exenteração posterior com cistectomia e ureterectomia parciais $(n=2)$. Resultados - A média de tempo cirúrgico foi de 403 minutos (280-485). A média de sangramento foi de $1620 \mathrm{~mL}$ (300-4800). A mortalidade pós-operatória foi de $6,66 \%(\mathrm{n}=1)$. A morbidade pós-operatória foi de 53,3\% $(\mathrm{n}=8)$. Os exames histológicos evidenciaram que todas as ressecções foram R0. Envolvimento linfonodal foi observado em quatro pacientes (26,66 \%) sendo que todos faleceram em decorrência da neoplasia. A sobrevida global em cinco anos foi de 35,7\%. Conclusão - A exenteração pélvica ainda apresenta alta morbidade, no entanto permanece justificada, pois pode conferir maior controle do câncer de reto $\mathrm{T} 4$ em longo prazo.

DESCRITORES - Exenteração pélvica. Neoplasias retais.

\section{INTRODUÇÃO}

A primeira exenteração pélvica foi reportada por Brunschwig no ano de 1948. Coube a esse autor a descrição da técnica que consistia na remoção de todos os órgãos pélvicos, tanto do aparelho reprodutor quanto do aparelho urinário, além do cólon sigmóide e reto com a realização de ureterosigmoidostomia ${ }^{(3)}$. BRICKER et al. ${ }^{(1)}$ melhoraram esse procedimento por meio do uso de um segmento ileal para confecção da derivação urinária, assim a ureteroileostomia cutânea era posicionada no lado direito do abdome, enquanto a colostomia permanecia à esquerda $\mathrm{a}^{(1,2,8)}$.

Enquanto a exenteração supra-elevadora preserva o assoalho pélvico, a exenteração infra-elevadora, por sua vez, envolve a ressecção da porção inferior da vagina, vulva ou períneo, incluindo o ânus e uretra. A exenteração supra-elevadora tem sua maior indicação para os tumores pélvicos que não envolvam o aparelho esfincteriano, podendo desta forma, estar associada a maiores índices de conservação esfincteriana. Em contrapartida, a exenteração infra-elevadora é habitualmente reservada para os casos em que ocorra o envolvimento dessas estruturas ou para casos de tumores pélvicos volumosos que impossibilitem uma abordagem exclusivamente por via abdominal ${ }^{(11)}$.
As exenterações pélvicas têm sido indicadas para o tratamento de diferentes neoplasias pélvicas, tanto primárias quanto residuais ou ainda mais raramente recurrentes. Embora historicamente tenham sido descritas para o tratamento do câncer de colo uterino avançado, essas operações têm sido preconizadas para o tratamento de tumores localmente avançados de reto, endométrio, vagina, vulva ou mesmo bexiga urinária. Em situações especiais, pode ser indicada ainda para o tratamento do câncer recurrente de ovário ou, excepcionalmente, para correção de fístulas complexas pós-radioterapia ${ }^{(7)}$.

Estima-se que até $5 \%$ dos tumores primários do reto apresentem aderências que envolvam a bexiga urinária, a próstata, os anexos ou a vagina. Em contrapartida, a própria distinção entre aderência ou invasão pode ser impossível, mesmo com julgamento intra-operatório minucioso. Desta maneira, somente a avaliação histológica do espécime ressecado, pode determinar com certeza, se ocorre ou não a invasão de estruturas contíguas ${ }^{(4,16)}$. Estudos prévios têm demonstrado que margens negativas obtidas com uma ressecção multivisceral fazem com que a recurrência local diminua significantemente de $69 \%$ para $18 \%$ e os índices de sobrevida por sua vez alcancem até $54,1 \%$ em 5 anos $^{(6,10)}$. IKE et al. ${ }^{(6)}$ inclusive, observaram sobrevida

Serviço de Cirurgia Geral - Oncocirurgia do Hospital do Servidor Publico Estadual de São Paulo - "Francisco Morato de Oliveira", São Paulo, SP.

Correspondência: Dr. Sergio Renato Pais Costa - Instituto de Oncologia São Paulo - Avenida Pacaembu, 1400 - 01234-000 - São Paulo, SP. E-mail: sergiorenatopais@ig.com.br 
em 10 anos de 50\% para esse grupo de doentes. Embora neste estudo a morbidade tenha sido significante $(66 \%)$, a mortalidade pós-operatória foi de somente $4,2 \%$.

Especificamente para tumores de reto T4 poucas séries de exenteração pélvica têm sido publicadas ${ }^{(5,6,13,17,20)} \mathrm{e}$, particularmente em nosso meio, ainda não foi relatada uma série exclusivamente de tumores de reto $\mathrm{T} 4$ cujos pacientes foram tratados com exenteração pélvica. A maioria das séries reportadas envolve amostras heterogêneas que reúnem desde recidivas pélvicas de câncer de reto, até mesmo casuísticas de outros tumores primários que apresentam prognósticos e resultados divergentes. Tal heterogeneidade da amostra pode acarretar falsas conclusões em relação aos resultados dessa operação ${ }^{(7,8,11,21)}$.

O objetivo do presente estudo foi avaliar os resultados a curto e longo prazo da exenteração pélvica no tratamento exclusivamente dos tumores de reto (adenocarcinoma) T4. Desta maneira, os autores conduziram uma análise retrospectiva dos tumores de reto T4 tratados por meio de exenteração pélvica no Hospital do Servidor Público Estadual de São Paulo - "Francisco Morato de Oliveira", São Paulo, SP. Foram avaliados: o tipo de exenteração, mortalidade, complicações, sobrevida em longo prazo, sobrevida livre de doença e modo de recurrência.

\section{MÉTODOS}

Foram estudados 15 casos de adenocarcinoma de reto T4 selecionados por meio do registro institucional de câncer do Hospital do Servidor Público Estadual de São Paulo, no período entre setembro de 1998 e junho de 2006. Todos os pacientes do presente estudo foram operados exclusivamente pelo Serviço de Cirurgia Geral e Oncocirurgia desse hospital. Apenas os tumores T4 que invadiam órgãos pélvicos contíguos foram considerados nesta casuística.

Dos 15 pacientes estudados, 8 eram do sexo masculino enquanto 7 eram do sexo feminino. A idade variou de 36 a 77 anos com média etária de 65 anos e mediana de 66 anos. Em relação à etnia, 11 eram caucasianos enquanto 5 eram da raça negra. Oito apresentavam alguma doença associada à neoplasia retal. Cinco apresentavam hipertensão arterial sistêmica (HAS) enquanto três apresentavam diabetes mellitus tipo II (DM). Apenas um apresentava obesidade.

Todos apresentavam sintomas incapacitantes. Sete apresentavam dois ou mais sintomas. Foram encontrados: dor pelve-perineal $(n=14)$, sangramento retal ou vaginal $(n=6)$, infecção urinária recurrente $(\mathrm{n}=3)$ e fístulas tanto retovaginal $(\mathrm{n}=1)$, quanto retovesical $(\mathrm{n}=1)$.

Todos tiveram o tumor diagnosticado por exames clínico, radiológico e histológico. Em 13 o tumor era localizado no reto médio ou baixo, enquanto em 2 , era localizado no reto alto.

Em relação ao grau de diferenciação do tumor, a distribuição foi: bem diferenciado em seis, moderadamente diferenciado em sete e pouco diferenciado em dois. As características demográficas, clínicas e histológicas estão demonstradas na Figura 1.

\begin{tabular}{|lc|}
\hline $\mathrm{n}=15$ & $65(36-77)$ anos \\
Média etária & 8 \\
\hline Sexo & 7 \\
$\quad$ Masculino & \\
Feminino & 5 \\
Doenças associadas & 3 \\
$\quad$ HAS & 1 \\
DM & \\
Obesidade & \\
& 14 \\
Sintomas & 6 \\
$\quad$ Dor & 3 \\
Sangramento & 2 \\
ITU recurrente & \\
Fístula & \\
& 13 \\
Localização & 2 \\
$\quad$ Reto médio-baixo & \\
Reto alto & 6 \\
Grau de diferenciação & 7 \\
Bem diferenciado & \\
Moderadamente diferenciado & \\
Pouco diferenciado & \\
\hline
\end{tabular}

HAS: hipertensão arterial sistêmica

DM: diabetes mellitus

TTU: infecço de trato urinário

FIGURA 1. Características clínicas e demográficas dos pacientes submetidos a exenteração pélvica por câncer de reto T4

$\mathrm{Na}$ presente casuística, apenas os pacientes que foram submetidos a algum tipo de exenteração pélvica padronizada foram considerados para estudo. Todos foram submetidos a colonoscopia com biopsia, tomografia computadorizada de abdome-pelve e raio-X simples de tórax para estádio. Também foram dosados os níveis séricos de CEA e CA 19,9. Cinco foram ainda submetidos a ultra-sonografia anorretal. Somente pacientes com tumor estádio T4 pela avaliação histológica completa do espécime cirúrgico foram considerados. Pacientes com tumor estádio T3 ou recidiva pélvica foram excluídos. Foram consideradas também apenas as ressecções potencialmente curativas nessa amostra.

Toda exenteração pélvica total (EPT) foi realizada conforme a técnica descrita por MORIYA et al. ${ }^{(13)}$, padronizada no Instituto Nacional do Câncer - Tókio, Japão. Os princípios técnicos seguidos foram: linfadenectomia ampliada com ligadura alta da artéria mesentérica inferior e para os casos de tumores abaixo da deflexão peritonial, dissecção linfonodal pélvica bilateral ao longo dos vasos ilíacos externos, incluindo também a fossa obturadora. Além disso, a remoção completa e em monobloco de todas as estruturas pélvicas como reto, bexiga, ureteres abaixo da bifurcação dos vasos ilíacos, órgãos genitais internos e vasos ilíacos internos bilaterais $^{(13)}$. Para as pacientes do sexo feminino que apresentavam tumores com comprometimento exclusivo do trato genital interno, a cirurgia preconizada foi a exenteração pélvica posterior (ressecção anterior do retossigmóide ampliada com uma histerectomia radical em monobloco associada à linfadenectomia pélvica).

Em relação ao tipo de exenteração pélvica, foram realizados: exenteração infra-elevadora $(n=5)$, exenteração infra-elevadora e sacrectomia parcial $(n=1)$, exenteração 
supra-elevadora $(n=4)$, exenteração posterior $(n=3)$ e, finalmente, exenteração pélvica posterior ampliada com cistectomia e ureterectomia parciais $(n=2)$. Houve conservação esfincteriana em sete pacientes (cinco exenterações posteriores e duas supra-elevadoras). Em relação ao tipo de derivação urinária, foram realizados: ureteroileostomia cutânea à Bricker $(\mathrm{n}=10)$ e ureteroneocistostomia à Boari $(\mathrm{n}=2)$. O tempo cirúrgico variou de 280 a 485 minutos, com mediana de 403 minutos. Dez pacientes foram transfundidos, sendo que o sangramento intra-operatório variou de 300 a $4.800 \mathrm{~mL}$ com mediana de $1.620 \mathrm{~mL}$. O tempo mediano de internação foi de 14 dias, variando de 7 a 28 dias. Em relação ao tratamento multidisciplinar foi realizada: radioterapia neo-adjuvante $(n=2)$, radioquimioterapia neo-adjuvante $(n=9)$, quimioterapia adjuvante $(n=5)$ e radioquimioterapia adjuvante $(n=1)$. Dois doentes foram exclusivamente operados.

Após o tratamento cirúrgico, todos pacientes foram seguidos com intervalos de 3 meses (até 2 anos), de 6 meses (entre 2 e 5 anos) e finalmente cada 12 meses (após 5 anos). A cada retorno, foram realizadas, além do exame físico completo, as dosagens de CEA e CA 19,9, ultra-sonografia total de abdome, tomografia computadorizada de abdomepelve e raios-X simples de tórax. Também foi realizada uma colonoscopia por ano, a partir do primeiro ano de cirurgia. Recurrência tumoral foi considerada somente em casos de documentação radiológica inequívoca e, se possível, com confirmação histológica.

\section{RESULTADOS}

Um paciente faleceu no período pós-operatório, o que acarretou uma mortalidade global de 6,66\%. Esse paciente, um indivíduo do sexo masculino com idade de 65 anos foi submetido a uma exenteração infra-elevadora com sacrectomia parcial. Evoluiu sem complicações até o $7^{\circ}$ dia pós-operatório, quando finalmente apresentou embolia pulmonar.

A morbidade global da presente série foi de 53,3\%. Oito pacientes apresentaram nove complicações. Somente um paciente foi submetido a reoperação, evoluindo com abcesso pélvico, drenado por laparotomia. As complicações pós-operatórias estão demonstradas na Tabela 1.

TABELA 1. Complicações e mortalidade

\begin{tabular}{lc}
\hline Morbidade & $53,3 \%(8)$ \\
\hline Complicações & 1 \\
Insuficiência renal aguda & 1 \\
Broncopneumonia & 1 \\
Íleo prolongado & 2 \\
Infecção urinária & 1 \\
Fístula retovaginal & 1 \\
Embolia pulmonar & 1 \\
Abcesso pélvico & $6,66 \%(1)$ \\
Reoperação & $6,66 \%(1)$ \\
Mortalidade &
\end{tabular}

Todas as ressecções foram R0 à avaliação histológica do espécime cirúrgico. Os órgãos mais comumente invadidos foram: bexiga $(n=12)$, próstata $(n=7)$, vesículas seminais $(n=3)$, ureter $(n=2)$, útero $(n=7)$, vagina $(n=3)$ e sacro $(\mathrm{n}=1)$. A mediana de linfonodos dissecados foi de 34 , variando entre 25 e 45 . Houve comprometimento linfonodal em quatro pacientes $(26,6 \%)$. Dois deles apresentaram comprometimento de linfonodos regionais (mesoreto), enquanto dois apresentaram comprometimento das cadeias da fossa obturadora (um paciente) e da ilíaca externa (um paciente), respectivamente.

Em relação aos sintomas pré-operatórios, apenas um paciente não atingiu o controle total após o procedimento cirúrgico e continuou utilizando derivados opiáceos para controle da dor; embora em menor dosagem e freqüencia. Todos os demais voltaram a suas atividades diárias e com controle total de seus sintomas após procedimento cirúrgico. O índice de sucesso de controle dos sintomas foi de $94,4 \%(n=14)$.

A mediana de seguimento foi de 48 meses (2-84 meses), com média de 43 meses. A sobrevida global em 5 anos foi de $35,7 \%$. A sobrevida em 5 anos dos pacientes sem envolvimento linfonodal em 5 anos foi de $45,45 \%$. Nesse grupo, oito pacientes sobreviveram pelo menos 36 meses (sobrevida máxima de 80 meses). Em contrapartida, nenhum paciente do grupo com envolvimento linfonodal sobreviveu 5 anos (sobrevida entre 13 a 28 meses). A sobrevida mediana do subgrupo sem envolvimento linfonodal foi de 48 meses, enquanto com envolvimento linfonodal foi de apenas 20 meses. Esses resultados estão demonstrados na Tabela 2 .

TABELA 2. Características do tempo de seguimento e sobrevida

\begin{tabular}{lc}
\hline Mediana de seguimento & $48(2-84)$ meses \\
\hline Sobrevida global em 5 anos & $35,7 \%$ \\
Sobrevida em 5 anos / linfonodo negativo & $45,45 \%$ \\
Sobrevida em 5 anos / linfonodo positivo & $0 \%$ \\
\hline
\end{tabular}

Ao todo seis pacientes apresentaram recidivas (40\%). Em relação a sua localização, os órgãos comprometidos foram: fígado $(\mathrm{n}=1)$, pelve $(\mathrm{n}=1)$, peritônio e fígado $(\mathrm{n}=2)$, peritônio e pelve $(n=1)$ e pulmão $(n=1)$. O intervalo livre de doença variou de 9 a 30 meses, com mediana de 20 meses. A sobrevida após recidiva variou de 3 a 7 meses, com mediana de 5 meses. Todos os pacientes com comprometimento linfonodal apresentaram algum tipo de recidiva e acabaram falecendo em decorrência da doença (a sobrevida variou de 13 a 28 meses). Em contrapartida, dos 11 pacientes sem comprometimento linfonodal apenas dois apresentaram recidivas (um paciente no fígado e outro no fígado e peritônio) em período que variou entre 20 e 30 meses. O paciente com recidiva hepática e peritonial permaneceu vivo até os 20 meses de seguimento (final do estudo), enquanto o paciente com recidiva hepática exclusiva faleceu em sua decorrência aos 36 meses de seguimento (realizou hepatectomia de resgate aos 18 meses após exenteração). Esses números estão demonstrados na Tabela 3. 
TABELA 3. Características das recidivas

\begin{tabular}{lc}
\hline Recidiva & $\mathrm{n}=6(40 \%)$ \\
\hline Fígado & 1 \\
Peritônio + fígado & 2 \\
Peritônio + pelve & 1 \\
Pelve & 1 \\
Pulmão & 1 \\
Mediana do intervalo livre de doença (meses) & $20(9-30)$ \\
Mediana de sobrevida/recidiva (meses) & $5(3-7)$
\end{tabular}

\section{DISCUSSÃO}

Um câncer retal é definido como T4 quando invade diretamente outros órgãos ou estruturas, e/ou perfura o peritônio ${ }^{(18)}$. Essa definição por sua vez, não condiz com a prática clínica diária em que a maioria dos autores considera que os verdadeiros tumores T4 são aqueles que invadem diretamente os outros órgãos pélvicos ${ }^{(13)}$. O tratamento desse grupo de pacientes é complexo e controverso. Para os tumores localmente avançados T4, diversos estudos têm demonstrado que tanto a radioquimioterapia quanto a radioterapia neo-adjuvantes podem conferir, respectivamente, aumento da ressecabilidade e maior sobrevida. Além disso, o tratamento neo-adjuvante também pode colaborar significantemente para a diminuição dos índices de recidiva pélvica. Desta maneira, para esse grupo de enfermos $(\mathrm{T} 4 / \mathrm{N}+)$ muitos autores têm preconizado algum tipo de neo-adjuvância. Embora essa tendência seja uma constante na literatura, em determinadas circunstâncias essa estratégia ou simplesmente não funciona ou não é factível. Diante dessas situações especiais, somente por meio de uma ressecção multivisceral, o paciente pode alcançar maior sobrevida ou até mesmo cura. Em contrapartida, apenas margens livres podem conferir esses objetivos e para que isso ocorra, a ressecção em monobloco deve ser priorizada. Além disso, a própria distinção entre aderências tumorais ou inflamatórias tanto no pré quanto no intra-operatório pode ser de difícil avaliação, restando assim, como alternativa tática, a ressecção multivisceral que permanece plausível e justificável nessa situação. Apesar do benefício, essas operações carregam altos índices de morbidade e deve-se, portanto, reservá-las tão somente a situações potencialmente curativas ${ }^{(13,14)}$.

Os índices de sobrevida em 5 anos dos pacientes com adenocarcinoma de reto localmente avançado $(\mathrm{T} 4 / \mathrm{N}+)$ e que são submetidos a algum tipo de exenteração pélvica, têm se situado em torno de $20 \%$ até $70 \%$. No entanto, a simples comparação dessas taxas pode ser equivocada, haja vista que devido a sua indicação restrita poucos serviços as têm realizado em grande número e, paralelamente, as próprias indicações têm sido diferentes dentro das diversas instituições ${ }^{(6)}$. IKE et al ${ }^{(6)}$ observaram taxas de sobrevida em 5 e 10 anos de respectivamente $54,1 \%$ e $50 \%$, resultado similar ao observado por MORIYA et al. ${ }^{(13)}$, que também no Japão reportaram índice de sobrevida em 5 anos de $57 \%$. No ocidente, poucos estudos foram realizados, todos inclusive com amostras pequenas como a da presente série. Dentre esses estudos, LASSER et al..$^{(9)}$ observaram uma sobrevida em 5 anos de $18 \%$ de 10 pacientes, enquanto LAW et al. ${ }^{(10)}$ observaram sobrevida em 5 anos de 54\% em 5 anos, em casuística de 15 pacientes. Mais recentemente, VERMASS et al. ${ }^{(19)}$ na Holanda, relataram sobrevida estimada em 5 anos de $52 \%$ para 23 pacientes com tumores de reto T4 submetidos a exenteração pélvica. Embora a presente casuística seja pequena, pelo menos cinco pacientes $(35,7 \%)$ sobreviveram por no mínimo 5 anos, reforçando assim, a tese de que mesmo com indicação restrita, a exenteração pélvica pode prolongar a sobrevida de doentes com câncer de reto T4 em casos selecionados.

Não obstante avanço, tanto da parte técnica quanto do suporte intra e pós-operatório, tenha ocorrido nas últimas décadas, a mortalidade pós-operatória ainda permanece considerável. Segundo IKE et al. ${ }^{(6)}$, os índices de mortalidade têm se situado entre $0 \%$ e 20,8\%. Esses índices têm variado principalmente em função do tipo de exenteração e da experiência do serviço. Em grandes centros, mais recentemente, tem se observado diminuição significativa da mortalidade, que tem se situado entre $1,6 \%$ a $4,2 \%{ }^{(6,13,19)}$. Embora a presente amostra seja pequena e o hospital não especializado em oncologia, um único paciente faleceu, justamente o que foi submetido a exenteração infra-elevadora com ressecção óssea (sacrectomia parcial). Sendo assim, a mortalidade global de $6,66 \%$ se situa dentro do reportado na literatura e condizente com esse tipo de cirurgia ultra-radical.

A exenteração pélvica, mesmo em centros especializados, tem envolvido treinamento intenso e exaustivo da equipe cirúrgica. Conhecimentos tanto das reconstruções complexas do sistema digestório (anastomoses baixas, bolsas) quanto do trato urinário (anastomoses, derivações e reservatórios urinários), têm sido peças fundamentais para o seu êxito. O tempo cirúrgico é geralmente extenso e quase sempre associado à hemorragia de grande monta, seja durante a operação ou mesmo durante o período pós-operatório, tornando assim, freqüente a necessidade de transfusões. Destarte, apenas pacientes altamente selecionados (hígidos) e com oportunidade real de cura devem ser eleitos para essa cirurgia. Pacientes com múltiplas doenças associadas, metástases à distância ou mesmo invasão de estruturas ósseas (coluna lombar, parede pélvica) e nervosas (nervo ciático, raízes sacrais) não têm sido considerados candidatos a essa operação. Paralelamente, envolvimento de vasos ilíacos internos ou mesmo obstrução ureteral bilateral, também têm sido considerados contra-indicações ${ }^{(16,17)}$.

Conquanto uma diminuição da mortalidade tenha ocorrido, o mesmo não tem sido observado em relação à morbidade que ainda permanece alta. Estudos recentes têm apontado morbidade entre $20 \%$ e $70 \%$ nas diferentes séries, com média em torno de $50 \%^{(4,5,6,10,14,16,17,18,20,21)}$. A morbidade de $53,3 \%$ observada na presente casuística, mesmo que oriunda de um hospital geral e de treinamento, encontra-se dentro do previsto. Essa morbidade, inclusive, é menor do que a relatada em estudos prévios de grandes centros especializados em oncologia ${ }^{(6,21)}$. Dentre as principais complicações relatadas, as deiscências de anastomoses tanto do trato digestivo quanto urinário e as infecciosas (do trato urinário, pneumonias), têm sido as mais comumente encontradas. Além disso, mais raramente, complicações circulatórias como tromboembolismo ou mesmo infarto agudo do miocárdio também têm sido relatadas. Ainda que a morbidade seja elevada e reflita em aumento do tempo de internação e dos custos de tratamento, 
ela não tem acarretado aumento do índice de reoperação, que permanece baixo ${ }^{(5,6,10,13,14,15,18,21)}$.

Diversos fatores prognósticos têm sido associados a um impacto negativo na sobrevida dos pacientes com tumor de reto localmente avançado. Dentre os mais comumente citados, o envolvimento linfonodal parece ser dos mais importantes ${ }^{(6,17)}$. Embora um teste estatístico específico não tenha sido realizado na presente amostra, todos pacientes que apresentaram envolvimento linfonodal acabaram por falecer de recidiva em um período máximo de 28 meses após a cirurgia. Em contrapartida, dentre os 11 pacientes sem linfonodos comprometidos, cinco deles (45\%) sobreviveram mais que 60 meses.

A maioria dos tumores de reto T4 condena os doentes a sofrimento atroz e debilitante, influenciando negativamente na sua qualidade de vida. A exenteração pélvica promove controle de maneira eficiente da maioria desses sintomas. Tanto as fístulas (retovaginais ou retovesicais) quanto o sangramento (vaginal ou retal) são os sintomas mais freqüentemente controlados pela cirurgia. No presente estudo houve melhora considerável dos sintomas em relação à condição pré-operatória. Embora não tenha sido utilizado um questionário específico para a avaliação da qualidade de vida, a maioria desses pacientes voltou a sua rotina diária, sem quaisquer dos sintomas incapacitantes observados no período pré-operatório. O controle da dor foi possível na maioria dos pacientes, contudo apenas dois experimentaram recidiva pélvica; assim não se pode concluir que a resolutividade a longo prazo da dor possa ser alcançada por meio dessa operação ${ }^{(4,7,17)}$.

\section{CONCLUSÃO}

A exenteração pélvica nas suas diferentes variações técnicas é procedimento cirúrgico de mortalidade considerável e alta morbidade. Em contrapartida, pode conferir maior sobrevida aos doentes com adenocarcinoma de reto localmente avançado (T4). Essa opção terapêutica deve ser considerada com reserva e deve ter sua indicação restrita a uma população altamente selecionada com intuito curativo. Além disso, pode oferecer melhoria da qualidade de vida desses enfermos que, freqüentemente, apresentam sintomas debilitantes e de difícil manejo clínico.

Costa SRP, Antunes RCP, Paula RP, Pedroso MA, Farah JFM, Lupinacci RA. Pelvic exenteration for T4 rectal cancer: a series of 15 ressectable cases. Arq Gastroenterol. 2007;44(4):284-8.

ABSTRACT - Background - Pelvic exenteration is the best therapeutic choice for treatment of T4 rectal cancer. Although, this operation still presents considerable mortality and high morbidity. Aim - To report on a series of 15 patients with a T4 rectal cancer at a general hospital and describe the outcomes (morbidity, mortality and long-term survival) following pelvic exenteration. Methods - Complete follow-up data were available on 15 patients who underwent pelvic exenteration for T4 rectal cancer between 1998 and 2006. These subjects comprised seven men and eight women with a mean age of 65 years. All of them presented serious incapacitating complaints. The surgical procedures included: infraelevator exenteration $(n=6)$, supraelevator exenteration $(n=4)$, posterior exenteration $(n=3)$ and, posterior exenteration plus partial cystectomy and ureterectomy $(n=2)$. Results - The mean duration of surgery was 403 minutes (280-485). The mean blood loss was $1620 \mathrm{~mL}(300-4.800)$. The postoperative mortality was $6,66 \%(\mathrm{n}=1)$. The overall rate morbidity was $53,3 \%(\mathrm{n}=8)$. The pathological examination showed that all resections were R0. Lymph node involvement was present in four patients (26,66 \%), and all of them died due to tumor recurrence. The overall 5-year survival rate was 35,7\%. Conclusion - In spite of its aggressive nature and high morbidity, pelvic exenteration seems justified in rectal carcinoma when the disease extends to the urinary or genital tract. This procedure may offer long-term disease control.

HEADINGS - Pelvic exenteration. Rectal neoplasms.

\section{REFERÊNCIAS}

1. Bricker EM. Bladder substitution after pelvic evisceration. Surg Clin North Am. 1950;30:1511-21.

2. Bricker EM, Butcher HR Jr, McAfee CA. Late results of bladder substitution with isolated ileal segments. Surg Gynecol Obstet. 1954;99:469.

3. Brunschwig A. Complete excision of pelvic viscera for advanced carcinoma. Cancer. 1948; 177-83

4. Church JM, Gibbs P, Chao MW, Tjandra JJ. Optimizing the outcome for patients with rectal cancer. Dis Colon Rectum. 2003;46:389-402.

5. Hida J, Yasutomi M, Maruyama T. Results from pelvic exenteration for locally advanced colorectal cancer with lymph node metastases. Dis Colon Rectum. 1998;41:165-8.

6. Ike H, Shimada H, Yamaguchi S, Ichikawa Y, Fujii S, Ohki S. Outcome of total pelvic exenteration for primary rectal cancer. Dis Colon Rectum. 2001;46:474-80.

7. Kecmanovic DM, Pavlov MJ, Kovacevic PA, Sepetkovski AV, Ceranic, Stamenkovic AB. Management of advanced pelvic cancer by exenteration. Eur J Surg Oncol. 2003;29:743-6

8. Kiselow M, Butcher Hr Jr, Bricker EM. Results of the radical surgical surgical treatmen of advanced pelvic cancer: a fifteen-year study. Ann Surg. 1967;166:428-36.

9. Lasser P, Doidy L, Elias D. Total pelvic exenteration and rectal cancer. Apropos of 20 cases. Chirurgie. 1999;124:252-7.

10. Law Wl, Chu KW, Choi HK. Total pelvic exenteration for locally advanced rectal cancer. J Am Coll Surg. 2000;190:78-83.

11. Magrina JF, Stanhope CR, Weaver AL. Pelvic exenterations: supraelevator, infraelevator, and with vulvectomy. Gynecol Oncol. 1997;64:130-5.
12. McKenzie SP, Barnes SL, Schwartz RW. An update on the surgical management of rectal cancer. Curr Surg. 2005;62:407-11.

13. Moriya Y, Akasu T, Fujita S, Yamamoto S. Agressive surgical treatment for patients with T4 rectal cancer. Colorectal Dis. 2003;5:427-31

14. Polk HG Jr. Extended resection for selected adenocarcinoma of the large bowel. Ann Surg. 1972:175:892-9.

15. Ruo L, Guillem JG. Surgical management of primary colorectal cancer. Surg Oncol. 1998; 7:153-63.

16. Sasson AR, Sigurdson ER. Management of locally advanced rectal cancer. Surg Oncol. 2000;9:193-204.

17. Shirouzu K, Isomoto H, Kakegawa T. Total pelvic exenteration for locally advanced colorectal carcinoma. Br J Surg. 1996;83:32-5.

18. UICC TNM. Colon and rectum. In: Sobin LH, Wittekind Ch, editors. Classification of malignant tumors. 5th ed. New York: Wiley-Liss; 1997. p. 67.

19. Vermaas M, Ferenschild FT, Verhoef C, Nuyteens JJ, Marinelli AW, Wiggers T, Kirkel WJ, Eggermont AM, Wilt JH. Total pelvic exenteration for primary locally advanced and locally recurrent rectal cancer. Eur J Surg Oncol. 2007;33:452-8.

20. Verschueren RC, Mulder NH, Hooykaas JA, Szabo BG, Karrenbeld A. Pelvic exenteration for advanced primary rectal cancer in male patients. Clin Oncol (R Coll Radiol). 1998;10:318-21.

21. Wydra D, Emerich J, Sawicki S, Ciachk K, Marciniak A. Major complications following exenteration in cases of pelvic malignancy: 10-year experience. World J Gastroenterol. 2006;12:1115-9.

Recebido em 3/11/2006 Aprovado em 8/6/2007 